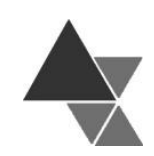

\title{
Desperdício de alimentos: fator de insegurança alimentar e nutricional
}

\author{
Sílvia Panetta Nascimento ${ }^{1}$
}

O crescimento demográfico projetado para as próximas décadas implicará na necessidade de maior produção de alimentos para alimentar cerca de 9 bilhões de pessoas que deverão existir no ano de 2050. As modernas técnicas de produção de alimentos, tanto na agropecuária como nos processos de beneficiamento e armazenagem, promoveram maior produtividade e eficiência dos sistemas produtivos. Esses sistemas, entretanto, vêm reduzindo a capacidade de recuperação dos solos, desregulação da dinâmica hídrica e redução da biodiversidade, o que pode pôr em risco a produção de alimentos, em função da escassez dos recursos naturais. Os recursos devem, portanto, ser preservados e muito bem utilizados, no entanto, diversos estudos demonstram que há alto grau de desperdício na cadeia de produção e consumo de alimentos. Neste artigo, por meio de uma revisão bibliográfica, propôs-se analisar se o desperdício de alimentos pode afetar a segurança alimentar e nutricional. A metodologia adotada foi a pesquisa qualitativa com análise documental, legislativa e bibliográfica. Concluiu-se que, embora sejam muitos os aspectos que interferem na segurança alimentar e nutricional, principalmente aqueles de caráter sócio econômico, as perdas na produção de alimentos consomem recursos naturais, os quais podem se tornar escassos e comprometer a produção e consumo de alimentos no futuro, gerando mais um fator de insegurança alimentar e nutricional.

Palavras-chave: produção de alimentos, sustentabilidade, recursos naturais, perdas.

\section{Food waste: a factor of food and nutritional insecurity}

Demographic growth projected for the coming decades will imply the need for increased food production to feed the approximately 9 billion people expected to exist by the year 2050. Modern food production techniques, both in agriculture and in processing and processing increased productivity and efficiency of production systems. These systems, however, have reduced the capacity of soil recovery, deregulation of water dynamics and reduction of biodiversity, which could put in risk the food production due to the scarcity of natural resources. The resources must therefore be preserved and very well used, however, several studies have shown that there is a high degree of waste in the food production and consumption chain. In this article, through a bibliographical review, it was proposed to analyze if food wastage can affect food and nutritional security. The methodology adopted was the qualitative research with documentary, legislative and bibliographic research. It was concluded that, although we have many aspects interfering in food security, especially those of a socio-economic nature, losses in food production consume natural resources, which may become scarce and engage food production and consumption in the future, generating another factor of food insecurity.

Keywords: food production, sustainability, natural resources, losses.

${ }^{1}$ Docente da Fatec Itapetininga - Centro Estadual de Educação Tecnológica Paula Souza e Coordenadora Científica na Higiene Alimentar Publicações e Serviços. Endereço para correspondência: Rua Saldanha Marinho no 125, sala 2, CEP 18200-001, Itapetininga, SP. Telefone: (15) 35271749. E-mail. silvia.nascimento@fatec.sp.gov.br 


\section{INTRODUÇÃO}

Conforme projeções da Organização das Nações Unidas para Agricultura e Alimentação $(\mathrm{FAO})^{[1]}$, estima-se que deverá haver um aumento de $60 \%$ na produção de alimentos a fim de atender uma população de 9 bilhões de pessoas em 2050.

O avanço tecnológico ocorrido no último século, com o desenvolvimento de tecnologias de produção, armazenamento e beneficiamento de alimentos, possibilita produzir o montante de alimentos necessário para atender ao crescimento da população projetado pela $\mathrm{FAO}$, diferentemente do que se acreditou em séculos passados, quando havia dúvidas se seria possível produzir alimentos necessários para atender a uma população em crescimento.

A falta de alimentos, entretanto, é uma realidade, mas ocorre por outras razões que não a dificuldade em produzir os alimentos. Estudos demonstram que esse é, principalmente, um problema socioeconômico causado pela má distribuição das riquezas ${ }^{[2]}$.

Por outro lado, mesmo com uma melhor distribuição, é preciso considerar que haverá ainda a necessidade de aumento do volume de alimentos produzidos para as próximas décadas ${ }^{[1]}$ e uma nova incerteza, quanto à possibilidade de atender a essa demanda, foi identificada: se os recursos naturais serão suficientes para atender à produção necessária

Os sistemas produtivos adotados nas últimas décadas vêm sendo questionados em função dos impactos ambientais que promoveram, trazendo desmatamento, erosão dos solos e desregulação da dinâmica hídrica, além de reduzirem a biodiversidade e, consequentemente, aumentarem a necessidade do uso de insumos e defensivos químicos. A agricultura, que é a base para toda a produção de alimentos, depende fundamentalmente desses recursos e sua escassez poderá comprometer todo o processo, promovendo, possivelmente, a falta dos alimentos necessários para atender às futuras demandas ${ }^{[3]}$.

Essa preocupação vem gerando reações e, atualmente, novos modelos produtivos que preservam melhor as condições ambientais, já vêm sendo adotados, como a rotação de culturas, a produção integrada, bem como os sistemas agroecológicos. Contudo, mesmo nesses sistemas, os recursos são necessários e, portanto, não podem ser desprezados. É isso o que ocorre, porém, quando existem perdas e desperdício dos alimentos produzidos. Estudos demonstram que a taxa de desperdício na produção de alimentos é da ordem de $20 \%{ }^{[4]}$ e ocorre em todas as etapas da cadeia de produção e consumo dos principais grupos de alimentos produzidos mundialmente ${ }^{1]}$.

Neste artigo propõe-se analisar, por meio de uma revisão bibliográfica sobre o tema, como o desperdício de alimentos pode afetar a segurança alimentar e nutricional. A metodologia adotada foi a pesquisa qualitativa com análise documental, legislativa e bibliográfica.

\section{Demanda por alimentos}

A fim de atender a uma população de 9 bilhões de pessoas em 2050, conforme apontam as projeções de crescimento demográfico, a FAO estima que deverá haver um aumento de $60 \%$ na produção de alimentos ${ }^{[5]}$. Esse aumento por si só não é uma dificuldade, haja vista que a evolução tecnológica verificada no século XX, possibilitou a multiplicação no volume de alimentos produzidos, com uma eficiência e velocidade sem precedentes. A disponibilidade dos recursos naturais para atender a esse crescimento na produção de alimentos, porém, constitui-se em extremo desafio ${ }^{[6]}$.

Em décadas passadas, já houve a preocupação com a disponibilidade de alimentos, quando a fome era uma realidade de grande parte da população mundial, conforme evidenciado por Josué de Castro, problema este agravado ao final da Segunda Guerra Mundial, o que levou a Organização das Nações Unidas (ONU) a incluir, na Declaração Universal dos Direitos Humanos (1948), em seu artigo XXV, o direito à alimentação[7].

Justificada pelo propósito de aumentar a produção de alimentos e erradicar a fome, a Revolução Verde trouxe um novo modelo de produção agrícola, intensificando a produção por meio da mecanização agrícola, uso maciço de fertilizantes químicos e seleção genética de espécies mais produtivas ${ }^{[8]}$. Esse modelo 
produtivo, entretanto, passou a ser duramente questionado em função dos impactos ambientais gerados pela sua prática[ ${ }^{[\oplus]}$.

O modelo agrícola advindo desse período e globalmente adotado privilegia a prática da monocultura e da seleção genética, o que trouxe impactos negativos à própria produção de alimentos, uma vez que tais práticas comprometem a biodiversidade. Esse modelo de produção também envolve desmatamento de grandes áreas e esgotamento do solo, o que gerou a necessidade do uso ampliado de fertilizantes químicos e de defensivos agrícolas, indispensáveis para combater as pragas decorrentes da uniformização das culturas que tende a gerar espécies resistentes e levar ao desaparecimento de outras que poderiam promover o controle biológico natural[3].

A adoção desse modelo agrícola por muitos anos, em determinadas áreas, vem colocando em risco a disponibilidade dos recursos naturais, principalmente em regiões tropicais onde a diversidade biológica é naturalmente maior e, consequentemente, os danos advindos dessa prática são também intensificados. Conforme apontado por Soares e Porto[?], " $90 \%$ da produção mundial de alimentos estão restritos a somente quinze espécies vegetais e oito animais". A simplificação dos ecossistemas, tende a se tornar insustentável a longo prazo, havendo a necessidade de uma mudança na forma de produção, de acordo com as conclusões dos referidos autores.

Apesar desse sistema de produção ter sido apresentado, em décadas passadas, como solução para o problema da fome do mundo, os impactos ambientais gerados por sua prática ameaçam a segurança alimentar e nutricional da população mundial, pondo em risco a própria disponibilidade dos alimentos, já que esta depende essencialmente dos recursos naturais ${ }^{[3]}$.

A questão que se apresenta é a necessidade do aumento da produção de alimentos para atender a uma população ainda em crescimento, mas considerando-se que o aumento da população já traz por si só impactos ao ambiente, com as mudanças climáticas, sobre uso de recursos, escassez de água, entre outros, os modelos de produção agrícolas atuais ainda acentuam esses problemas, colocando em risco a segurança alimentar e nutricional, mesmo que esta não dependa apenas da quantidade de alimentos, mas também e, principalmente de sua distribuição igualitária[ ${ }^{[]}$.
Um aspecto importante que contribui para agravar a disponibilidade mundial de alimentos é o elevado padrão de perdas. Estudos indicam que o desperdício na produção de alimentos é expressivo, podendo atingir, até $2050,25 \%$ do volume global de alimentos produzidos. Essas perdas ocorrem em toda a cadeia produtiva, no entanto, as maiores taxas são observadas nas etapas de distribuição, reduzindo a vantagem do empenho em obter maior produtividade ${ }^{[10]}$.

\section{Desperdício na produção de alimentos}

Muitas vezes usados como sinônimos, os termos perdas e desperdício apresentam diferenças intrínsecas em suas definições. Como estabelece a $\mathrm{FAO}^{[11]}$, a perda de alimentos (food loss) refere-se à redução do volume ou do valor nutricional (qualidade) dos alimentos produzidos para consumo humano, enquanto desperdício (food waste) abrange o rejeito de alimentos. Também os principais dicionários da língua portuguesa fazem diferenciações entre os termos. No dicionário Aurélio[ ${ }^{[2]}$, por exemplo, perdas são definidas como danos, prejuízos e desperdício como uma despesa inútil e censurável. Mesmo reconhecendo que ambos deveriam ser evitados, é possível perceber, nessas definições, uma certa crítica ao termo desperdício, apresentado como uma ação prejudicial e até pouco ética. Não que as perdas também não o sejam, mas aqui cabe apresentar as causas dessas ações.

Para a FAO[11], as perdas são causadas principalmente pela ineficiência nas cadeias de abastecimento, ressaltando-se falhas na infraestrutura, logística e gestão, além de desastres naturais, portanto, não são intencionais. Já o desperdício é consequência do excesso da oferta em um determinado mercado consumidor ou por hábitos de consumo, o que pode ocasionar perdas por deterioração ou expiração do prazo de validade. Desperdício, portanto, compreende perdas, mas trata-se do descarte intencional dos alimentos.

Conforme o relatório Os Rastros do Desperdício de Alimentos: Impactos sobre os Recursos Naturais, da Organização das Nações Unidas para Alimentação e Agricultura- $\mathrm{FAO}\left[{ }^{[13]}\right.$, são desperdiçados anualmente 1,3 bilhões de toneladas de alimentos, volume este que, além de causar elevadas perdas econômicas, também compromete os recursos naturais, os quais são 
essenciais para que a humanidade continue a produzir alimentos.

O estudo Losses, inefficiencies and waste in the global food system, recém-publicado na Agricultural Systems ${ }^{4]}$, registra que, no mundo, cerca de $20 \%$ dos alimentos disponibilizados são perdidos. Essas perdas foram calculadas em diferentes etapas do sistema agroalimentar, sendo consideradas, no estudo, seis categorias: agricultura, pecuária, armazenamento e transporte, processamento, desperdício no consumo e excesso de consumo.

O relatório da $\mathrm{FAO}^{[11]}$, também considera níveis distintos de perdas conforme a etapa da cadeia agroalimentar, somando-se $54 \%$ de desperdício na produção agrícola e pós colheita, enquanto as etapas de processamento, distribuição e consumo, respondem por $46 \%$.
Esses valores tendem a sofrer variações conforme o nível de desenvolvimento dos países, apresentando-se diversamente nas regiões de renda média e elevada, onde o desperdício na distribuição e consumo tende a ser maior ${ }^{[13]}$, conforme registrado na Figura 1.

Em estudo publicado pela $\mathrm{FAO}^{[1]}$ em 2011, foram apresentadas estimativas de perdas alimentares ao longo da cadeia de produção e consumo, para os principais grupos de alimentos. Os dados para a América Latina estão reunidos no Quadro 1 e são coerentes com outros estudos já realizados sobre essa temática[10].

Quadro 1. Estimativa de perdas, em porcentagem, para grupos de alimentos por etapa da cadeia produtiva na América Latina

\begin{tabular}{|l|c|c|c|c|c|}
\hline & $\begin{array}{c}\text { Produção } \\
\text { agropecuária }\end{array}$ & $\begin{array}{c}\text { Pós colheita e } \\
\text { armazenamento }\end{array}$ & $\begin{array}{c}\text { Processamento e } \\
\text { embalagem }\end{array}$ & Distribuição & $\begin{array}{c}\text { Consumo } \\
\text { doméstico }\end{array}$ \\
\hline Cereais & 6,0 & 4,0 & 2,0 a 7,0 & 4,0 & 10,0 \\
\hline Raízes e tubérculos & 14,0 & 14,0 & 12,0 & 3,0 & 4,0 \\
\hline Oleaginosas e leguminosas & 6,0 & 3,0 & 8,0 & 2,0 & 2,0 \\
\hline Frutas e vegetais & 20,0 & 10,0 & 20,0 & 12,0 & 10,0 \\
\hline Carnes & 5,3 & 1,1 & 5,0 & 5,0 & 6,0 \\
\hline Pescados & 5,7 & 5,0 & 9,0 & 10,0 & 4,0 \\
\hline Leite & 3,5 & 6,0 & 2,0 & 8,0 & 4,0 \\
\hline
\end{tabular}

Fonte: $\mathrm{FAO}(2011)^{[1]}$

Figura 1. Desperdício de alimentos (contribuição relativa), por região e por etapa da cadeia produtiva.

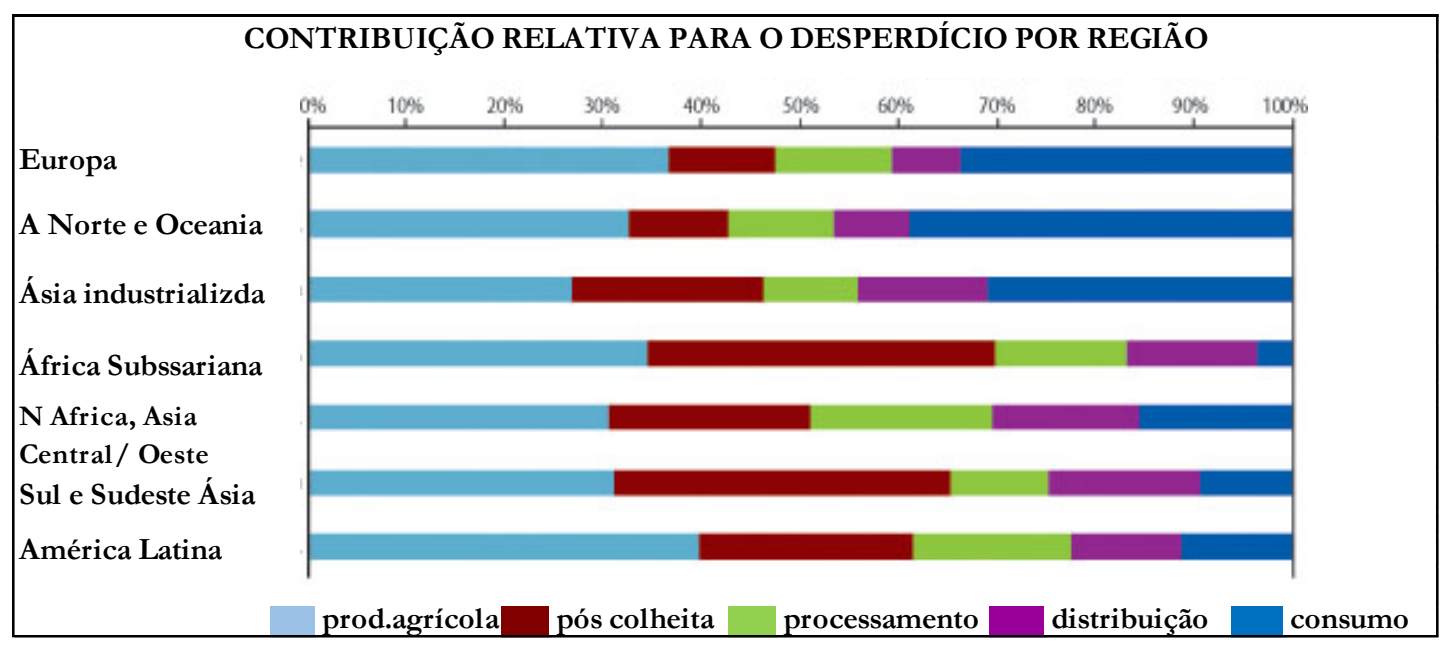


Alimentos sem valor comercial, nem sempre perderam seu valor nutritivo e, portanto, poderiam ainda ser utilizados, trazendo benefícios tanto para os produtores, que não teriam os custos do descarte na redução dos lucros, como para os consumidores que teriam maior oferta de produtos de qualidade, possivelmente até com preços mais baixos, enquanto os consumidores que se encontram à margem do mercado poderiam ter acesso a alimentos sem valor comercial, mas com qualidade nutritiva ${ }^{[10]}$.

No atendimento dos requisitos nutricionais de uma população em crescimento, as perdas, decorrentes de um sistema agroindustrial pouco eficiente, interferem negativamente na produção sustentável de alimentos ${ }^{[4]}$.

\section{Segurança alimentar e sustentabilidade}

A preocupação com a segurança alimentar, em nível internacional, foi formalizada ao final da 1a Guerra Mundial, quando foi considerada indispensável, para obtê-la, a capacidade dos países em produzir alimentos. Posteriormente verificou-se que, além da oferta de alimentos, era necessário, ainda, o acesso da população aos mesmos, de modo que, em 1986, a Cúpula Mundial de Alimentação estabeleceu:

\footnotetext{
“A segurança alimentar, nos níveis individual, familiar, nacional, regional e global, é alcançada quando todas as pessoas têm, a todo momento, acesso físico e econômico a alimentos inócuos e nutritivos para satisfazer suas necessidades dietéticas e preferencias alimentares para uma vida ativa e saudável[14].
}

O conceito de segurança alimentar abrange diversos aspectos, como a qualidade e inocuidade dos alimentos produzidos, a variedade que atenda os hábitos e a cultura alimentar, assim como a sustentabilidade do sistema alimentar. Considerando-se a definição de segurança alimentar, portanto, há que garantir a oferta e o acesso aos alimentos no presente, sem, contudo, comprometer a capacidade futura de produção[15].

Esse é justamente o conceito de desenvolvimento sustentável, conforme foi definido no relatório da ONU de 1987, intitulado Nosso Futuro
Comum, "O desenvolvimento sustentável é o desenvolvimento que encontra as necessidades atuais sem comprometer a habilidade das futuras gerações de atender suas próprias necessidades". Em consequência dessas disposições ficou reconhecida em todo o mundo a relação entre o desenvolvimento e o meio ambiente, bem como a necessidade de adotar um modelo sustentável de desenvolvimento econômico ${ }^{[16]}$.

Desde então, diversas Conferências e ações vem sendo realizadas em todo o mundo e, em 2015, na Cúpula de Desenvolvimento Sustentável, os governos dos países participantes desse evento estabeleceram os objetivos do desenvolvimento sustentável, entre os quais se propõem a erradicar a fome e a má nutrição. $O$ $12 \circ$ objetivo estabelece "assegurar padrões de produção e consumo sustentáveis" [17].

As considerações sobre desenvolvimento sustentável realizadas nos fóruns mundiais repercutiram na formação de políticas nacionais, tendo o Brasil assumido crescente importância nesse debate, principalmente ao sediar eventos relevantes como a Conferência das Nações Unidas sobre Meio Ambiente e Desenvolvimento (Rio92) e a Conferência das Nações Unidas sobre Desenvolvimento Sustentável $(\text { Rio }+20)^{[18] .}$

Em relação à segurança alimentar, entretanto, foi apenas na década de 80 que a temática foi incorporada às políticas públicas do país. O conceito de segurança alimentar, no Brasil, formalizou-se na I Conferência Nacional de Alimentação e Nutrição, realizada em 1986[19] e, em 2004, durante a II Conferência Nacional de Segurança Alimentar e Nutricional, foi aprovada a definição de Segurança Alimentar e Nutricional, a qual foi incorporada à Lei Orgânica de Segurança Alimentar[20].

Nesse documento já foi estabelecida uma relação de interdependência entre a produção de alimentos e a sustentabilidade, segundo consta no artigo 3o da referida Lei:

Segurança Alimentar e Nutricional (SAN) é a realização do direito de todos ao acesso regular e permanente a alimentos de qualidade, em quantidade suficiente, sem comprometer o acesso a outras necessidades essenciais, tendo como base práticas 
alimentares promotoras de saúde, que respeitem a diversidade cultural e que sejam social, econômica e ambientalmente sustentáveis ${ }^{[20]}$.

O parágrafo II do artigo 4o da Lei Orgânica estabelece que "a segurança alimentar e nutricional abrange a utilização sustentável dos recursos"[20], portanto, conforme apresentado por Costa e Bogus ${ }^{[21]}$, o campo da segurança alimentar e nutricional compreende as políticas de combate ao desperdício e aos sistemas que degradam o ambiente.

Ainda, a Política Nacional de Segurança Alimentar e Nutricional, tem como uma de suas diretrizes a "promoção do abastecimento e estruturação de sistemas sustentáveis e descentralizados, de base agroecológica, de produção, extração, processamento e distribuição de alimentos"[20].

Entende-se, portanto, que em todas as etapas da produção de alimentos, o desperdício é fator de insegurança alimentar e nutricional, uma vez que sua prática é contrária aos sistemas sustentáveis de produção.

É importante ressaltar, entretanto, que, embora a quantidade de alimentos descartados no mundo seja suficiente para alimentar a população que passa fome, não é possível estabelecer uma relação direta entre o fim do desperdício e a ausência de fome, pois a principal causa da fome ainda é a dificuldade de acesso aos alimentos ${ }^{[2]}$.

\section{CONSIDERAÇÕES FINAIS}

Os fatores que geram insegurança alimentar e nutricional são vários mas, atualmente, assume papel de destaque principalmente, fatores sócio econômicos gerados pela má distribuição das riquezas e, consequentemente, falta de acesso aos alimentos, causando a fome de milhares de indivíduos em todo o mundo.

Apenas produzir mais alimentos, portanto, não irá resolver o problema da fome e insegurança alimentar e nutricional, no entanto, a falta de alimentos, em quantidade, pode sim comprometer a segurança alimentar e nutricional e, uma vez que se estima ser necessário aumentar o volume de alimentos nas próximas décadas para alimentar uma população ainda em crescimento, serão necessários recursos naturais para atender a essa demanda.

Os recursos, entretanto, já se encontram comprometidos em função dos impactos ambientais gerados pelos sistemas produtivos adotados nas últimas décadas e, mesmo que existam reações a esses impactos com a adoção de modelos mais sustentáveis, ainda há a necessidade do uso de recursos, os quais são frequentemente desperdiçados em função das perdas que ocorrem em toda a cadeia de produção de alimentos, gastando recursos que não se converterão em alimentos utilizáveis.

Estudos demonstram que o desperdício de alimentos atinge a cifra de $20 \%$ do volume produzido, gerando produtos que, além de não atingirem o fim a que foram destinados, interferem na eficiência da cadeia produtiva, trazendo prejuízos para produtores e consumidores. Em função das perdas, produz-se mais do que seria necessário, utilizando-se recursos desnecessariamente, o que poderá comprometer a produção no futuro, quando haverá maior demanda, afetando a segurança alimentar e nutricional.

Mesmo considerando que apenas a redução do desperdício não irá, necessariamente, melhorar a segurança alimentar e nutricional, já que esta é também uma questão de acesso, o desperdício pode afetar os recursos e, consequentemente, reduzir a oferta de alimentos e, neste caso, afetar negativamente a segurança alimentar e nutricional.

\section{REFERÊNCIAS}

[1] FAO. Global food losses and food waste - Extent, causes and prevention. Rome: FAO; 2011.

[2] Brasil. Conselho Nacional de Segurança Alimentar e Nutricional. Estudo destaca desperdício de alimentos no mundo [Internet]. 2017 [acesso em 10 abr 2017]. Disponível em http://www4.planalto.gov.br/consea/comunicacao/noticias/2017 /estudo-destaca-desperdicio-de-alimentos-no-mundo

[3] Zimmermann CL. Monocultura e transgenia: impactos ambientais e insegurança alimentar. Veredas do Direito. 2009;6(12):79-100.

[4] Alexander P, Brown C, Arneth A, Finnigan J, Moran D, Rounsevell MDA. Losses, inefficiencies and waste in the global food system. Agricultural Systems. 2017;153:190-200. 
[5] Mutela H. O crescimento populacional e a questão alimentar. O País [Internet]. 2014 [acesso em 10 mai 2017]. Disponível em http://www.fao.org

[6] Brauner MCC, Graff L. Segurança alimentar e produção agrícola: reflexões sobre a ótica da justiça ambiental. Veredas do Direito. 2015;12(24):375-400.

[7] ONU. Declaração Dos Direitos Humanos [Internet]. 1948 [acesso em 10 mai 2017]. Disponível em http://www.onu.org.br

[8] Albergoni L, Pelaez V. Da Revolução Verde à agrobiotecnologia: ruptura ou continuidade de paradigmas? Revista de Economia. 2007;33(1):31-53.

[9] Soares WL; Porto MF. Atividade agrícola e externalidade ambiental: uma análise a partir do uso de agrotóxicos no cerrado brasileiro. Ciênc. Saúde Coletiva. 2007;12(1):131-143

[10] Belik W, Cunha ARAA, Costa LA. Crise dos alimentos e estratégias para a redução do desperdício no contexto de uma política de Segurança alimentar e nutricional no Brasil. Planejamento e Políticas Públicas. 2012;38:107-132.

[11] FAO. Food Wastage Footprint Impacts on natural resources. Summary Report. [Internet]. Rome; 2013 [acesso em 15 abr 2017]. Disponível em http://www.fao.org

[12] Aurélio - Dicionário de Português Online [Internet]. 2017 [acesso em 10 mai 2017]. Disponível em https:/ /dicionariodoaurelio.com

[13] FAO. Desperdício de Alimentos tem consequências no clima, na água, na Terra e na biodiversidade [Internet]. 2017 [acesso em 10 abr 2017]. Disponível em http://www.fao.org.br

[14] Nunes, MF. O direito fundamental à alimentação [dissertação]. São Paulo: Pontifícia Universidade Católica de São Paulo; 2006.

[15] Maluf RS, Menezes F. Caderno “Segurança Alimentar'. Agência Embrapa de Informação Tecnológica [Internet]. 2017 [acesso em 15 out 2017]. Disponível em: http:/ /www.agencia.cnptia.embrapa.br

[16] Nações Unidas no Brasil. A ONU e o meio ambiente [Internet]. 2017 [acesso em 10 out 2017]. Disponível em: https://nacoesunidas.org/acao/meio-ambiente

[17] Jardim A, Machado Filho JV, Oliveira MRM. Segurança alimentar na era da sustentabilidade. RMAI 125. Economia Verde. 2017;(1):05-15.

[18] Brasil. Itamaraty. O Brasil e o desenvolvimento sustentável [Internet]. 2017 [acesso em 10 set 2017]. Disponível em http://www.itamaraty.gov.br

[19] Belik W. A Política Brasileira de Segurança Alimentar e Nutricional: concepção e resultados. Segur. Aliment. Nutr. 2012;19(2):94-110.
[20] Brasil. Lei no 11.346, de 15 de setembro de 2006. Cria o Sistema Nacional de Segurança Alimentar e Nutricional - SISAN com vistas em assegurar o direito humano à alimentação adequada e dá outras providências. Diário Oficial da União. 18 set 2006.

[21] Costa CA, Bogus CM. Significados e Apropriações da Noção de Segurança Alimentar e Nutricional pelo Segmento da Sociedade Civil do Conselho Nacional de Segurança Alimentar e Nutricional. Saude Soc. 2012;21(1):103-114. 